\title{
A uniqueness result for a simple force-free magnetic field submitted to a topological constraint
}

\author{
J. J. Aly \\ CEA/DSM/DAPNIA, Service d'Astrophysique (CNRS FRE 2591), CE Saclay, 91191 Gif-sur-Yvette Cedex, France \\ e-mail: jjaly@discovery.saclay.cea.fr \\ Received 29 June 2004 / Accepted 13 August 2004

\begin{abstract}
A proof is given of the following statement: if $\boldsymbol{B}$ is a smooth force-free magnetic field contained in a cylindrical domain of axis parallel to $\hat{z}$ and of star-shaped cross-section, and if $\boldsymbol{B}$ is topologically equivalent to the uniform field $\boldsymbol{B}_{0}=B_{0} \hat{z}$, then $\boldsymbol{B}=\boldsymbol{B}_{0}$. In addition to being a very first step in the general study of the uniqueness of a magnetostatic equilibrium subject to a topological constraint, this result has a direct relevance to the approach recently developed by $\mathrm{Ng} \&$ Bhattacharjee (1998, Phys. Plasma, 5, 4028) to support Parker's theory of current sheets formation in the solar corona.
\end{abstract}

Key words. magnetohydrodynamics (MHD) - Sun: magnetic fields - Sun: corona

\section{Introduction}

In many astrophysical situations (solar or stellar corona, magnetosphere of a disk-accreting compact object, ...), one has to deal with a magnetic field occupying a domain $D$ filled up with a tenuous perfectly conducting plasma and evolving quasistatically through a series of force-free configurations $\boldsymbol{B}(t)$ as a result of slow motions imposed to its footpoints on the boundary $\partial D$ (see, e.g., Aly 1994). A question which thus naturally arises is the following one: is there at any given time $t$ another equilibrium $\boldsymbol{B}^{\prime}(t)$ reachable from $\boldsymbol{B}(t)$ by some process compatible with the frozen-in law - in other words, is there a forcefree field $\boldsymbol{B}^{\prime}(t)$ topologically equivalent to $\boldsymbol{B}(t)$ in that sense that its magnetic lines can be obtained from those of the latter by a continuous deformation which does not move the footpoints on $\partial D$ ? Although this problem may look at first sight a little bit academic, there are actually at least two reasons why astrophysicists should undertake its study. Firstly, the existence of a $\boldsymbol{B}^{\prime}(t)$ with a lower energy would imply that $\boldsymbol{B}(t)$ be metastable, and thus able to suffer (if finitely perturbed) a catastrophic transition with an energy release - a flare. Secondly, as pointed out by the referee, modern observations of the solar corona magnetic field give now access to the connectivity of its lines (outlined, e.g., by TRACE), in addition to the values of its normal component on the photosphere (measured, e.g., by MDI on SOHO), and it would be quite helpful to know if there is only one force-free configuration compatible with a set of data of this type.

As a first little step towards an understanding of the quite difficult question just raised, we would like to consider in this Research Note the simplest possible situation. We take $D$ to be a finite cylinder of axis parallel to $\hat{z}$, the field to be the uniform $\boldsymbol{B}_{0}=B_{0} \hat{z}$, and we address the possible existence in $D$ of another force-free field topologically equivalent to $\boldsymbol{B}_{0}$. In fact, this problem has already been considered by $\mathrm{Ng} \&$ Bhattacharjee (1998, NB hereafter) as a part of an interesting argument designed to support Parker's well known idea according to which current sheets develop spontaneously when $\boldsymbol{B}_{0}$ is deformed by moving its footpoints (Parker 1979, 1994, and references therein). In NB, however, the field is treated in the framework of the reduced magnetohydrodynamics approximation - which eliminates from the very beginning the possibility of dealing with too large deformations of $\boldsymbol{B}_{0}-$, while we keep here the fully nonlinear equations for a force-free field, and we thus hope that our results may be also useful to strengthen NB's argument. There is actually another (but much less essential) difference between our work and NB: in the latter, the field occupies all the space $D_{\infty}$ between two parallel planes, where it is imposed to be doubly-periodic in $x$ and $y$, while we have chosen to work for mathematical convenience in the bounded cylindrical part $D$ of $D_{\infty}$.

This Note is organized as follows. We give in Sect. 2 a detailed description of our assumptions. Thus we develop our proof in three steps. In Sect. 3, we use arguments based on current conservation to derive constraints on the form of the integral of $B$ along a magnetic line. In Sect. 4, we establish (using in particular the well-known virial theorem) two integral relations satisfied by $\boldsymbol{B}$. And finally we combine in Sect. 5 the results of the two preceding sections to get our conclusion.

\section{Assumptions and equations}

We use Cartesian coordinates $(x, y, z)$, and decompose any vector $\boldsymbol{X}$ according to $\boldsymbol{X}=\boldsymbol{X}_{\perp}+X_{z} \hat{z}$. In particular, we write $\boldsymbol{r}=\boldsymbol{r}_{\perp}+z \hat{z}$ for the position vector $\boldsymbol{r}$. We consider a 


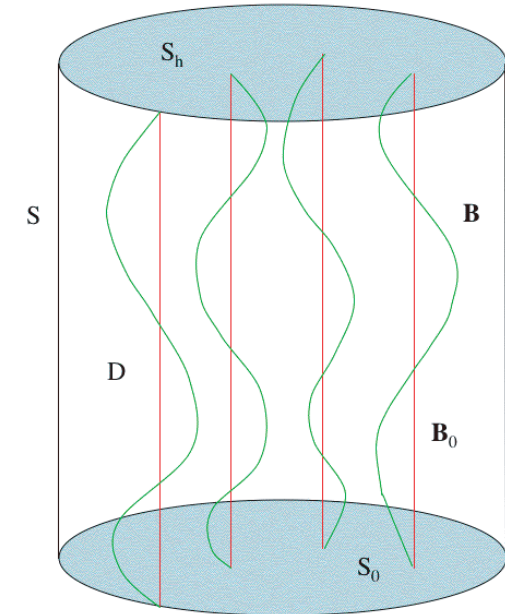

Fig. 1. The force-free field $\boldsymbol{B}_{0}$ (red lines) and the hypothetical forcefree field $\boldsymbol{B}$ with the same topology (green lines) occupying the cylinder $D$ bounded by the horizontal surfaces $S_{0}$ and $S_{\mathrm{h}}$ and the vertical surface $S$.

cylindrical domain $D$ of axis parallel to $\hat{z}$. The boundary $\partial D$ of $D$ is constituted of the "vertical" surface $S$ and the "horizontal" bounded surfaces $S_{0}$ and $S_{\mathrm{h}}$ located respectively in the planes $\{z=0\}$ and $\{z=h>0\}$ (see Fig. 1). The outer normal to $\partial D$ is denoted by $\hat{\boldsymbol{n}}$. The shape of $S_{0}$ (of course identical to that of $S_{\mathrm{h}}$ ) is left quite arbitrary. It is only imposed to be such that

$\boldsymbol{r}_{\perp} \cdot \hat{\boldsymbol{N}} \geq 0 \quad$ on $\partial S_{0}$,

where $\partial S_{0}$ and $\hat{N}$ denote, respectively, the curve bounding $S_{0}$ and its external normal in the plane $\{z=0\}$. A plane domain satisfying Eq. (1) is said to be star-shaped with respect to the origin $\mathrm{O}$ of $\boldsymbol{r}_{\perp}$.

We assume here that $D$ contains a smooth magnetic field $\boldsymbol{B}$ satisfying the following conditions:

- $\boldsymbol{B}$ is force-free, whence

$\boldsymbol{\nabla} \cdot \boldsymbol{B}=0$

$\boldsymbol{\nabla} \times \boldsymbol{B}=\alpha \boldsymbol{B}$,

where $\alpha(\boldsymbol{r})$ is a scalar function which is seen at once to satisfy $\boldsymbol{B} \cdot \nabla \alpha=0$, and is then constant along the lines of $\boldsymbol{B}$.

- $\boldsymbol{B}$ is topologically equivalent to the uniform field $\boldsymbol{B}_{0}=B_{0} \hat{z}$ (with $B_{0}>0$ a constant). As indicated in the introduction, we mean by this statement that the magnetic lines of $\boldsymbol{B}$ can be obtained from those of $\boldsymbol{B}_{0}$ by a smooth deformation keeping fixed the footpoints of the lines on $S_{0}$ and $S_{\mathrm{h}}$ (see Fig. 1). This assumption implies in particular that the normal component $B_{\mathrm{n}}=\boldsymbol{B} \cdot \hat{\boldsymbol{n}}$ of $\boldsymbol{B}$ on $\partial D$ does satisfy $B_{\mathrm{n}}=B_{0}$ on $S_{\mathrm{h}}, B_{\mathrm{n}}=-B_{0}$ on $S_{0}$ and $B_{\mathrm{n}}=0$ on $S$. Equivalently, we have $b_{\mathrm{n}}=\boldsymbol{b} \cdot \hat{\boldsymbol{n}}=0$ on $\partial D$ if we effect the decomposition

$\boldsymbol{B}=\boldsymbol{B}_{0}+\boldsymbol{b}$.

Physically, a field $\boldsymbol{B} \neq \boldsymbol{B}_{0}$ satisfying the conditions above if there is one - could possibly be produced by the following process, in which $D$ is taken to be filled up with a perfectly conducting plasma (see NB): (i) $\boldsymbol{B}_{0}$ is made to evolve quasistatically through a sequence of force-free fields up to some ideally unstable configuration $\boldsymbol{B}_{1}$ by imposing to its footpoints on $S_{\mathrm{h}}$, say, to move at the given velocity $\boldsymbol{v}(x, y)$ during a time $t_{1}$; (ii) the footpoints being kept fixed, $\boldsymbol{B}_{1}$ relaxes to a new stable equilibrium $\boldsymbol{B}_{1}^{\prime}$ of lower energy, the excess energy being radiated away by some arbitrary mechanism; (iii) $\boldsymbol{B}_{1}^{\prime}$ is set into a reverse quasi-static force-free evolution by applying to its footpoints on $S_{\mathrm{h}}$ the velocity field $-\boldsymbol{v}(x, y)$ during the time $t_{1}$, which of course bring back the footpoints to their initial positions, producing eventually $\boldsymbol{B}$.

\section{Consequences of current conservation}

Our topological assumption on the force-free field $\boldsymbol{B}$ may be explicited as follows: (i) a magnetic line $\mathcal{L}$ starting at $(x, y, 0)$ ends up at $(x, y, h)$; (ii) a line $\mathcal{L}$ does not enlace an other line $\mathcal{L}^{\prime}$. In particular, a line drawn on $S$ cannot go around $D$ one or several times. We derive here some analytic relations expressing necessary conditions for (i) and (ii) to be fulfilled.

We first consider a magnetic line $\mathcal{L}(x, y)$ starting at $(x, y, 0)$. By condition (i) and the conservation of $\alpha$ along $\mathcal{L}$, we have

$\alpha(x, y, h)=\alpha(x, y, 0)$.

Therefore, the vector $\beta$ defined on $S_{0}$ by

$\boldsymbol{\beta}(x, y)=\boldsymbol{b}_{\perp}(x, y, 0)-\boldsymbol{b}_{\perp}(x, y, h)$

does satisfy

$\nabla_{\perp} \times \boldsymbol{\beta}=[\alpha(x, y, 0)-\alpha(x, y, h)] B_{0} \hat{z}=0$,

which implies the existence on $S_{0}$ of a potential $\psi(x, y)$, defined up to an additive constant, such that

$\beta=\nabla_{\perp} \psi$.

Next we choose an arbitrary oriented open curve $C_{0}$ on $S_{0}$, with endpoints $\left(x_{1}, y_{1}, 0\right)$ and $\left(x_{2}, y_{2}, 0\right)$, and construct the magnetic surface $\Sigma$ formed of all the magnetic lines starting from $C_{0} . \Sigma$ is limited upstairs by the vertical projection $C_{h}$ of $C_{0}$ onto $S_{\mathrm{h}}$, and on the lateral sides by the two magnetic lines $\mathcal{L}_{1}=\mathcal{L}\left(x_{1}, y_{1}\right)$ and $\mathcal{L}_{2}=\mathcal{L}\left(x_{2}, y_{2}\right)$. With $\mathcal{C}_{0}, \mathcal{L}_{2},-\mathcal{C}_{h}$ and $-\mathcal{L}_{1}$ (the lines $\mathcal{L}$ being oriented by $\boldsymbol{B}$ ), we form the closed contour $C$ which is the oriented boundary of $\Sigma$, and we choose on the latter the normal $\hat{\boldsymbol{n}}$ in the usual way. Using successively the fact that $\boldsymbol{B}$. $\hat{\boldsymbol{n}}=0$ on $\Sigma$ (as the latter is a magnetic surface), Eq. (3), Stokes theorem, and Eqs. (6) and (8), we obtain

$$
\begin{aligned}
0 & =\int_{\Sigma} \alpha \boldsymbol{B} \cdot \hat{\boldsymbol{n}} \mathrm{d} s=\int_{\Sigma}(\boldsymbol{\nabla} \times \boldsymbol{B}) \cdot \hat{\boldsymbol{n}} \mathrm{d} s \\
& =\int_{\mathcal{C}_{0}} \boldsymbol{b}_{\perp} \cdot \mathrm{d} \boldsymbol{l}+\int_{\mathcal{L}_{2}} \boldsymbol{B} \cdot \mathrm{d} \boldsymbol{l}-\int_{\mathcal{C}_{h}} \boldsymbol{b}_{\perp} \cdot \mathrm{d} \boldsymbol{l}-\int_{\mathcal{L}_{1}} \boldsymbol{B} \cdot \mathrm{d} \boldsymbol{l} \\
& =\int_{\mathcal{L}_{2}} B \mathrm{~d} l-\int_{\mathcal{L}_{1}} B \mathrm{~d} l+\int_{\mathcal{C}_{0}} \boldsymbol{\beta} \cdot \mathrm{d} \boldsymbol{l} \\
& =\left[\int_{\mathcal{L}_{2}} B \mathrm{~d} l+\psi\left(x_{2}, y_{2}\right)\right]-\left[\int_{\mathcal{L}_{1}} B \mathrm{~d} l+\psi\left(x_{1}, y_{1}\right)\right] .
\end{aligned}
$$

Owing to the arbitrariness of $\left(x_{1}, y_{1}\right)$ and $\left(x_{2}, y_{2}\right)$, we conclude from the latter relation that the quantity $\int_{\mathcal{L}(x, y)} B \mathrm{~d} l+\psi(x, y)$ is 
independant of $(x, y)$ and we write

$\int_{\mathcal{L}(x, y)} B \mathrm{~d} l+\psi(x, y)=B_{0} h$

which fixes the gauge of $\psi$.

Finally, we consider a magnetic line $\mathcal{L}$ drawn on the vertical part $S$ of the boundary, which is by assumption a magnetic surface. We take the closed contour $C$ constituted of $-\mathcal{L}$ and of the vertical straight line starting from the lower footpoint of $\mathcal{L}$, and note that $C$ does limit a part $\Sigma$ of $S$ owing to condition (ii) above. As we have $(\boldsymbol{\nabla} \times \boldsymbol{B}) \cdot \hat{\boldsymbol{n}}=\alpha B_{\mathrm{n}}=0$ on $\Sigma \subset S$, we obtain by applying once more Stokes theorem and by taking into account Eq. (10)

$$
\begin{aligned}
\int_{0}^{h} B_{z}(x, y, z) \mathrm{d} z & =B_{0} h+\int_{0}^{h} b_{z}(x, y, z) \mathrm{d} z \\
& =\int_{\mathcal{L}(x, y)} B \mathrm{~d} l=-\psi(x, y)+B_{0} h,
\end{aligned}
$$

whence

$\int_{0}^{h} b_{z}(x, y, z) \mathrm{d} z+\psi(x, y)=0$

for $(x, y) \in \partial S_{0}$.

It may be worth noticing here that condition (i) - and a fortiori its consequences Eqs. (8) and (10) - cannot be sufficient to get uniqueness of the equilibrium $\boldsymbol{B}$. In fact, it is quite easy to construct an infinite family of equilibria $\left\{\boldsymbol{B}_{k}\right\}(k \in \mathbb{Z}, k \neq 0)$ satisfying (i) - but otherwise not topologically equivalent to $\boldsymbol{B}_{0}$ as it is necessary to move the footpoints on $S_{\mathrm{h}}$ to deform the lines of $\boldsymbol{B}_{0}$ into those of $\boldsymbol{B}_{k}$. Take indeed the field $\boldsymbol{B}_{0}$, and, the frozen-in law being enforced, produce some field $\boldsymbol{B}_{k}^{\prime}$ with an overall twist of all the lines by rotating an integer number $k$ of times all the upper footpoints around some point of $S_{\mathrm{h}}$. Next, fix the positions of the footpoints of $\boldsymbol{B}_{k}^{\prime}$ and let it relax to an equilibrium (e.g., by giving some viscosity to the plasma as in Moffat 1985): this leads to the seeked force-free field $\boldsymbol{B}_{k}$. The role of Eq. (12) is to eliminate all the fields of this type from our considerations. This equation does not apply indeed to $\boldsymbol{B}_{k}$, as a line of the latter drawn on $S$ makes one or several turns around $D$ when going from $S_{0}$ to $S_{\mathrm{h}}$, and $\mathcal{L}$ and the vertical line joining the two footpoints do no longer limit a part of $S$. Consequently, Stokes theorem cannot be applied anymore as done above.

\section{Integral relations}

\subsection{Magnetic energy}

We first note that

$\int_{D} b^{2} \mathrm{~d} v=\int_{D} B^{2} \mathrm{~d} v-\int_{D} B_{0}^{2} \mathrm{~d} v$

The term $2 B_{0} b_{z}$ in $B^{2}=B_{0}^{2}+2 B_{0} b_{z}+b^{2}$ integrates indeed to 0 over $D$ as the integral of $b_{z}$ over any horizontal cross section vanishes by flux conservation. Next, we evaluate as follows the first integral in the right-hand side of Eq. (13). We decompose $D$ into elementary flux tubes $\mathrm{d} \mathcal{T}$ constructed around the magnetic lines $\mathcal{L}$ of $\boldsymbol{B}$ and carrying a flux $\mathrm{d} \phi$, and note that

$$
\int_{\mathrm{d} \mathcal{T}} B^{2} \mathrm{~d} v=\int_{\mathcal{L}} B\left(B \mathrm{~d} s_{\mathrm{n}}\right) \mathrm{d} l=\mathrm{d} \phi \int_{\mathcal{L}} B \mathrm{~d} l,
$$

where $\mathrm{d} s_{\mathrm{n}}$ is the area of a normal cross section of $\mathrm{d} \mathcal{T}$ and $\mathrm{d} l$ is the element of length along $\mathcal{L}$. By summing over all the tubes and by writing $\mathrm{d} \phi=B_{0} \mathrm{~d} s$, where $\mathrm{d} s$ is the area of the intersection of $\mathrm{d} \mathcal{T}$ with $S_{0}$, we thus get

$$
\begin{aligned}
\int_{D} b^{2} \mathrm{~d} v & =B_{0} \int_{S_{0}}\left(\int_{\mathcal{L}} B \mathrm{~d} l-B_{0} h\right) \mathrm{d} s \\
& =-B_{0} \int_{S_{0}} \psi \mathrm{d} s,
\end{aligned}
$$

the second line being obtained by using Eq. (10) in the first one.

\subsection{The virial theorem}

Being a force-free field, $\boldsymbol{B}$ satisfies the virial theorem (see, e.g., Aly 1984)

$$
\int_{D} B^{2} \mathrm{~d} v=\int_{\partial D}\left[(\boldsymbol{r} \cdot \hat{\boldsymbol{n}}) B^{2}-2(\boldsymbol{r} \cdot \boldsymbol{B}) B_{\mathrm{n}}\right] \mathrm{d} s .
$$

The right-hand side of Eq. (16) can be transformed by noticing that: (i) on $S_{\mathrm{h}}, \hat{\boldsymbol{n}}=\hat{\boldsymbol{z}}, \boldsymbol{r} \cdot \hat{\boldsymbol{n}}=h, B_{\mathrm{n}}=B_{0}, B^{2}=B_{0}^{2}+b_{\perp}^{2}$, and $\boldsymbol{r} \cdot \boldsymbol{B}=\boldsymbol{r}_{\perp} \cdot \boldsymbol{b}_{\perp}+h B_{0}$; (ii) on $S_{0}, \hat{\boldsymbol{n}}=-\hat{z}, \boldsymbol{r} \cdot \hat{\boldsymbol{n}}=0, B_{\mathrm{n}}=-B_{0}$, and $\boldsymbol{r} \cdot \boldsymbol{B}=\boldsymbol{r}_{\perp} \cdot \boldsymbol{b}_{\perp} ;$ (iii) on $S, \boldsymbol{r} \cdot \hat{\boldsymbol{n}}=\boldsymbol{r}_{\perp} \cdot \hat{\boldsymbol{n}}, B^{2}=B_{0}^{2}+2 B_{0} b_{z}+b^{2}$, and $B_{\mathrm{n}}=0$. This leads to

$$
\begin{aligned}
\int_{D} B^{2} \mathrm{~d} v= & \int_{S_{\mathrm{h}}}\left[-h B_{0}^{2}+h b_{\perp}^{2}-2\left(\boldsymbol{r}_{\perp} \cdot \boldsymbol{b}_{\perp}\right) B_{0}\right] \mathrm{d} s \\
& +\int_{S_{0}} 2\left(\boldsymbol{r}_{\perp} \cdot \boldsymbol{b}_{\perp}\right) B_{0} \mathrm{~d} s \\
& +\int_{S}\left(\boldsymbol{r}_{\perp} \cdot \hat{\boldsymbol{n}}\right)\left(B_{0}^{2}+2 B_{0} b_{z}+b^{2}\right) \mathrm{d} s
\end{aligned}
$$

This relation is valid in particular for the equilibrium field $\boldsymbol{B}=\boldsymbol{B}_{0}(\boldsymbol{b}=0)$, giving

$$
\int_{D} B_{0}^{2} \mathrm{~d} v=-\int_{S_{\mathrm{h}}} h B_{0}^{2} \mathrm{~d} s+\int_{S}\left(\boldsymbol{r}_{\perp} \cdot \hat{\boldsymbol{n}}\right) B_{0}^{2} \mathrm{~d} s .
$$

Substracting Eqs. (18) from (17) and using Eq. (13) and the relation

$$
\begin{aligned}
-2 \int_{S_{\mathrm{h}}}\left(\boldsymbol{r}_{\perp} \cdot \boldsymbol{b}_{\perp}\right) B_{0} \mathrm{~d} s+ & 2 \int_{S_{0}}\left(\boldsymbol{r}_{\perp} \cdot \boldsymbol{b}_{\perp}\right) B_{0} \mathrm{~d} s \\
& =2 B_{0} \int_{S_{0}} \boldsymbol{r}_{\perp} \cdot \boldsymbol{\beta} \mathrm{d} s
\end{aligned}
$$

resulting from Eq. (6), we obtain finally

$$
\begin{aligned}
\int_{D} b^{2} \mathrm{~d} v= & h \int_{S_{\mathrm{h}}} b_{\perp}^{2} \mathrm{~d} s+\int_{S}\left(\boldsymbol{r}_{\perp} \cdot \hat{\boldsymbol{n}}\right) b^{2} \mathrm{~d} s \\
& +2 B_{0}\left(\int_{S}\left(\boldsymbol{r}_{\perp} \cdot \hat{\boldsymbol{n}}\right) b_{z} \mathrm{~d} s+\int_{S_{0}} \boldsymbol{r}_{\perp} \cdot \boldsymbol{\beta} \mathrm{d} s\right) .
\end{aligned}
$$




\section{The uniqueness theorem}

As a last step, we use the relations derived in Sect. 3 to evaluate the large parenthesis in the right-hand side of Eq. (20). Using Eq. (12), we get for the first term

$$
\begin{aligned}
\int_{S}\left(\boldsymbol{r}_{\perp} \cdot \hat{\boldsymbol{n}}\right) b_{z} \mathrm{~d} s & =\int_{\partial S_{0}}\left(\boldsymbol{r}_{\perp} \cdot \hat{\boldsymbol{N}}\right)\left(\int_{0}^{h} b_{z} \mathrm{~d} z\right) \mathrm{d} l \\
& =-\int_{\partial S_{0}}\left(\boldsymbol{r}_{\perp} \cdot \hat{\boldsymbol{N}}\right) \psi \mathrm{d} l
\end{aligned}
$$

where we have noted that

$\left(\boldsymbol{r}_{\perp} \cdot \hat{\boldsymbol{n}}\right)(x, y, z)=\left(\boldsymbol{r}_{\perp} \cdot \hat{\boldsymbol{N}}\right)(x, y)$ on $S$.

As for the second term, we get by using successively Eq. (8), Gauss theorem in the plane, and Eq. (15),

$$
\begin{aligned}
\int_{S_{0}} \boldsymbol{r}_{\perp} \cdot \boldsymbol{\beta} \mathrm{d} s & =\int_{S_{0}} \boldsymbol{r}_{\perp} \cdot \nabla_{\perp} \psi \mathrm{d} s \\
& =\int_{\partial S_{0}}\left(\boldsymbol{r}_{\perp} \cdot \hat{\boldsymbol{N}}\right) \psi \mathrm{d} l-2 \int_{S_{0}} \psi \mathrm{d} s \\
& =\int_{\partial S_{0}}\left(\boldsymbol{r}_{\perp} \cdot \hat{\boldsymbol{N}}\right) \psi \mathrm{d} l+\frac{2}{B_{0}} \int_{D} b^{2} \mathrm{~d} v .
\end{aligned}
$$

Injecting both results into Eq. (20) thus leads eventually to

$h \int_{S_{\mathrm{h}}} b_{\perp}^{2} \mathrm{~d} s+\int_{S} b^{2}\left(\boldsymbol{r}_{\perp} \cdot \hat{\boldsymbol{n}}\right) \mathrm{d} s+3 \int_{D} b^{2} \mathrm{~d} v=0$,

which implies

$\boldsymbol{b} \equiv 0 \quad$ in $D$

as $\boldsymbol{r}_{\perp} \cdot \hat{\boldsymbol{n}} \geq 0$ on $S$ by Eqs. (22) and (1) (note that this is the only place in our proof where assumption (1) is used). Therefore $\boldsymbol{B}=\boldsymbol{B}_{0}$. QED.

\section{Conclusion}

In this paper, we have given a rigorous proof of the following result: the only smooth force-free magnetic field topologically equivalent to the uniform field $\boldsymbol{B}_{0}=B_{0} \hat{z}$ in a cylindrical domain of axis parallel to $\hat{z}$ and of star-shaped cross-section, is the field $\boldsymbol{B}_{0}$ itself. Although this result may look modest and a priori evident, it is not completely trivial to prove (for the least, we have not been able to coin a simpler proof). In any case, it is the first one of this type we are aware of, and we may hope that the techniques we have introduced can be used to treat more general situations. In particular, we have started considering the case where the force-free equilibrium is no longer the simple $\boldsymbol{B}_{0}$, but is a field obtained from the latter by imposing given displacements $\boldsymbol{\xi}$ to the footpoints on $\partial D$. The relations given here have been generalized to that situation, and some evidences have been obtained in favor of the uniqueness of the equilibrium when the values of $|\nabla \boldsymbol{\xi}|$ (or alternatively $|\alpha|$ ) are not too large. But there is still a missing element to conclude in a rigorous way. Some extensions to more realistic configurations - like closed loops or arcades in a half-space - have also been undertaken, and we currently guess that uniqueness is likely to occur in that case too as long as the field is nearly potential - a complete proof of this statement being however still to be coined.

An other interesting question is the possibility of deriving rigorous uniqueness results when the equilibrium problem for $\boldsymbol{B}$ is formulated in the framework of reduced magnetohydrodynamics as in NB. In that case, it seems difficult at first sight to proceed as we did it here. This question is also under investigation, and some results will be reported soon.

Acknowledgements. I thank A. Bhattacharjee for kindly reading the manuscript, and an anonymous referee for suggestions as to how to improve of the paper.

\section{References}

Aly, J. J. 1984, ApJ, 283, 349

Aly, J. J. 1994, in Cosmical Magnetism, ed. D. Lynden-Bell (Cambridge: Pub. of the Inst. of Astronomy), 7

Moffat, H. K. 1985, J. Fluid Mech., 159, 359

Ng, C. S., \& Bhattacharjee, A. 1998, Phys. Plasmas, 5, 4028 (NB)

Parker, E. N. 1979, Cosmical Magnetic Fields (Oxford: Clarendon Press)

Parker, E. N. 1994, Spontaneous Current Sheets in Magnetic Fields (Oxford: Oxford University Press) 